\section{The Antinuclear Antibody Test in the Diagnosis of Antisynthetase Syndrome and Other Autoimmune Myopathies}

\section{To the Editor:}

Autoantibodies directed to intracellular macromolecules are a characteristic feature of antinuclear antibody (ANA)-associated rheumatic diseases (AARD) such as systemic lupus erythematosus, systemic sclerosis, mixed connective tissue disease, Sjögren syndrome, and autoimmune inflammatory myopathies (AIM). Some of these autoantibodies are highly specific for the individual AARD and hence are termed disease-specific antibodies, and some are included in the classification and/or diagnostic criteria for these diseases ${ }^{1}$. In this context, the report by Aggarwal, et al in The Journal ${ }^{2}$ is timely and provides insight into the value of screening for anticytoplasmic antibodies (anti-CytAb) as an adjunct to the diagnosis of the antisynthetase syndrome, a subset of $\mathrm{AIM}^{3}$. In their retrospective analysis of 202 patients with antisynthetase syndrome, the anti-CytAb test performed better than the conventional ANA indirect immunofluorescence (IIF) test in identifying antisynthetase syndrome as evidenced by higher sensitivity ( $72 \%$ vs $50 \%$ ) and specificity ( $89 \%$ vs $6 \%$ ). Consequently, they indicate that a negative ANA test does not rule out the diagnosis of antisynthetase syndrome or AIM.

That a negative ANA does not indicate autoantibody negativity in antisynthetase syndrome or AIM is an important message that may be misunderstood by clinicians. Despite remarkable efforts to standardize ANA nomenclature by the International Consensus on ANA Pattern (ICAP) committee ${ }^{4,5}$, there is considerable confusion about the term ANA. The efforts of the ICAP were recognized by Aggarwal, et al, but they, as well as some diagnostic laboratories and clinicians, continue to interpret and understand the definition of ANA in a strict sense, meaning only autoantibodies that react with nuclear targets are properly regarded as ANA. The spectrum of autoantibody targets in the nucleus is wide and includes components of the nuclear envelope, nuclear pore complex, nuclear matrix, nucleoplasm, chromatin and chromosomes, nucleolus, and promyelocyte leukemia/coiled bodies $^{1}$.

The ICAP group learned that in addition to laboratories that report anti-CytAb as a negative ANA, others generate separate reports indicating the presence of "true" ANA and/or antibodies to "other" (i.e., mitotic spindle, cytoplasmic, cell surface) reactivity, and some report a positive ANA when cytoplasmic components are reactive, such as mitochondria, cytoskeleton, GW/P bodies, endosomes, Golgi complex, endoplasmic reticulum, or the cytosol ${ }^{6}$. In considering this lack of standardized ANA reporting, the ICAP committee considered alternative nomenclature, such as anticellular antibodies, but after recognizing that the ANA terminology is entrenched in the literature, agreed to continue to use the term ANA, intending it to refer to the spectrum of intracellular components 5 . Nevertheless, in some jurisdictions' existing guidelines, reimbursement schedules and classification criteria are based on restricting ANA reporting to nuclear patterns ${ }^{4}$. In this regard, it is imperative that the clinician is informed whether their diagnostic laboratory reports reactivity only to nuclear components. If it does, then in the context of AIM and antisynthetase syndrome the clinician should request a test and a report that specifies the presence or absence of anti-CytAb. In addition, now that many of the IIF patterns have achieved international consensus, each pattern can be identified by descriptive terminology (i.e., cytoplasmic fine speckled as in the case of antisynthetase syndrome), and to ensure understanding by both the clinicians and diagnostic laboratory, this pattern might also be reported as pattern AC-20 (www.anapatterns.org/trees.php). Aggarwal, et al simplified their reporting to positive or negative and restricted their recognition of anti-CytAb IIF patterns to "speckled" or "diffuse," neither of which are included in the 9 different cytoplasmic patterns (AC15-23) specified by ICAP.

A second issue is the serum dilution used to detect ANA/anti-CytAb. The serum dilution of $1 / 40$ was chosen to increase the sensitivity of detecting anti-CytAb, with specific attention to characteristic antisynthetase syndrome autoantibodies $^{3}$. It is uncertain whether this is an advisable approach because by increasing the assay sensitivity to detect anti-CytAb in antisynthetase syndrome, there is a well-known concurrent loss of specificity (i.e., specificity of ANA was only 6\%). An international study by 34 experts on ANA testing concluded that the screening dilution should be defined locally, but an abnormal ANA should be set at the titer above the 95th percentile of a healthy control population, and in general, a screening dilution of 1/160 on conventional HEp-2 substrates is suitable for the detection of ANA in adult populations being evaluated for $\mathrm{AARD}^{7}$. In fairness, other studies ${ }^{8}$ published prior to more modern microscope optics, light sources, and reagents suggested screening at dilutions of both $1 / 40$ and $1 / 160$. Hence, adoption of a 1/40 dilution for antisynthetase syndrome depends on these and related technical factors, a point not clarified by Aggarwal, et al.

Another reason to reconsider ANA IIF testing as a screen for antisynthetase syndrome or AIM is that, despite years of efforts, the ANA test has been troubled by lack of accuracy and specificity. In the broader spectrum of newer autoantibodies related to AIM, IIF staining of HEp-2 substrates has not been reported as a good screening test. For example, in a study of a small cohort of sera containing anti-PL-7 autoantibodies, fewer than $50 \%$ were positive by IIF $^{9}$. In addition, by decreasing the screening dilution for IIF testing, nonspecific staining due to heterophile antibodies seen in a variety of infectious diseases, especially Epstein-Barr virus, can be a confounding factor in interpretation of positive IIF staining ${ }^{10}$

Other reasons for variable ANA IIF results include the expertise of the technologist reading the IIF patterns, the substrate, and/or the kit manufacturer. The study by Aggarwal, et al used the HEp-2000 substrate (ImmunoConcepts), which is engineered to detect anti-SSA/Ro60 autoantibodies by transfecting the cells with the cognate cDNA and overexpressing the Ro60 target ${ }^{11}$. Therefore, other diagnostic laboratories may not obtain equivalent results because the performance of HEp-2 cell substrates from different suppliers varies significantly in sensitivity for anti-CytAb, as shown, for example, by IIF studies of anti-ribosomal $\mathrm{P}$ antibodies ${ }^{12}$.

In effect, Aggarwal, et al suggest a separate approach to ANA testing for antisynthetase syndrome and interstitial lung disease (ILD), an approach that may be difficult to implement unless the payers are willing to accept another test fee item in their reimbursement schedules. The main issue, however, is that if the ANA at a serum dilution of $1 / 40$ performed on HEp-2000 cells as a screen for antisynthetase syndrome is positive or negative, then testing for specific antisynthetase syndrome autoantibodies on a multianalyte array platform such as addressable laser bead assays and line immunoassays is likely to be requested anyway, a dilemma appreciated by the ICAP ${ }^{4}$. Therefore, the cost of the anti-CytAb IIF approach may not be as "inexpensive and quick" as Aggarwal, et al suggest. Many of the AIM variants share clinical features and a definitive diagnosis is often difficult, especially when overlapping features of AARD are present (i.e., weakness, fatigue, arthritis, ILD, elevated creatine kinase $)^{13,14}$. In the clinical setting of AIM, where the diagnosis of antisynthetase syndrome is not clear and other conditions (i.e., infections, malignancies) are considered in the differential diagnosis, the efficiency, speed of results, and cost-effectiveness of using autoantigen arrays ${ }^{15}$, which include the most common AIM targets, should be considered as the serology test of first choice.

Historically, there have been challenges in developing and adopting classification criteria for antisynthetase syndrome and AIM, and only recently has there been more emphasis on the inclusion of myositis-specific autoantibodies (reviewed in Lundberg, et $a l^{16}$ and Senécal, et $a l^{17}$ ). Even then, prospective, multicenter studies are still required to validate these criteria. Eventually, the diagnosis of AIM often rests on muscle pathology, muscle magnetic resonance imaging, and the presence of other clinical features such as ILD. Aggarwal, et al raise the importance of ILD as a preceding or concomitant feature of antisynthetase syndrome, and as they point out, ILD can be associated with antisynthetase syndrome-related autoantibodies, even in the absence of clinically apparent myopathy. However, the spectrum of autoantibodies in ILD is also remarkably diverse ${ }^{18}$ and ILD is seen in the wider spectrum of $\mathrm{AIM}^{19}$ where the ANA, by any definition, may be negative.

Personal non-commercial use only. The Journal of Rheumatology Copyright $(\subset) 2018$. All rights reserved. 
Here we acknowledge that the standardization of autoantibody assays, especially those for myositis-specific antibodies, remains poor ${ }^{20}$. In addition, a significant challenge is the regulatory status of autoantibody assays for AIM. While outside the United States line immunoassays or dot blots are available for the detection of autoantibodies in AIM, there is currently no test available that is approved by the US Food and Drug Administration (FDA), and most laboratories rely on laboratory-developed tests. Although the ANA test is FDA-approved, the detection of anti-CytAb as an aid in the diagnosis of antisynthetase syndrome (as intended use) is not cleared by the FDA.

The study by Aggarwal, et al is an important addition to the literature. However, until studies are conducted that compare the anti-CytAb IIF approach for antisynthetase syndrome to AIM profiles based on multianalyte arrays, it remains speculative whether anti-CytAb will represent a useful or cost-effective approach for diagnostic laboratories or clinicians.

MARVIN J. FRITZLER, MD, PhD, Cumming School of Medicine, University of Calgary; MAY Y. CHOI, MD, Cumming School of Medicine, University of Calgary, Calgary, Alberta, Canada; MICHAEL MAHLER, PhD, Inova Diagnostics Inc., Division of Research, San Diego, California, USA. Dr. Fritzler is a consultant to Inova Diagnostics Inc. (San Diego) and Werfen International (Barcelona, Spain). Dr. Mahler is an employee of Inova Diagnostics Inc., a company that manufactures and sells autoantibody assays. Address correspondence to Dr. M.J. Fritzler, University of Calgary, 3330 Hospital Dr. NW, Calgary, Alberta T2N 4N1, Canada.E-mail: fritzler@ucalgary.ca

\section{REFERENCES}

1. Mahler M, Meroni PL, Bossuyt X, Fritzler MJ. Current concepts and future directions for the assessment of autoantibodies to cellular antigens referred to as anti-nuclear antibodies. J Immunol Res 2014;2014:315179.

2. Aggarwal R, Dhillon N, Fertig N, Koontz D, Qi Z, Oddis CV. A negative antinuclear antibody does not indicate autoantibody negativity in myositis: role of anticytoplasmic antibody as a screening test for antisynthetase syndrome. J Rheumatol 2017;44:223-9

3. Mahler M, Miller FW, Fritzler MJ. Idiopathic inflammatory myopathies and the anti-synthetase syndrome: a comprehensive review. Autoimmun Rev 2014;13:367-71.

4. Damoiseaux J, von Mühlen CA, Garcia-De La Torre I, Carballo OG, de Melo Cruvinel W, Francescantonio PL, et al. International consensus on ANA patterns (ICAP): the bumpy road towards a consensus on reporting ANA results. Auto Immun Highlights 2016;7:1.

5. Chan EK, Damoiseaux J, de Melo Cruvinel W, Carballo OG, Conrad K, Francescantonio PL, et al. Report on the second International Consensus on ANA Pattern (ICAP) workshop in Dresden 2015. Lupus 2016;25:797-804.

6. Stinton LM, Eystathioy T, Selak S, Chan EK, Fritzler MJ. Autoantibodies to protein transport and messenger RNA processing pathways: endosomes, lysosomes, Golgi complex, proteasomes, assemblyosomes, exosomes, and GW bodies. Clin Immunol 2004; 110:30-44.
7. Agmon-Levin N, Damoiseaux J, Kallenberg C, Sack U, Witte T, Herold M, et al. International recommendations for the assessment of autoantibodies to cellular antigens referred to as anti-nuclear antibodies. Ann Rheum Dis 2014;73:17-23.

8. Tan EM, Feltkamp TE, Smolen JS, Butcher B, Dawkins R, Fritzler MJ, et al. Range of antinuclear antibodies in "healthy" individuals. Arthritis Rheum 1997;40:1601-11.

9. Hervier B, Uzunhan Y, Hachulla E, Benveniste O, Nunes H, Delaval $\mathrm{P}$, et al. Antisynthetase syndrome positive for anti-threonyl-tRNA synthetase (anti-PL7) antibodies. Eur Respir J 2011;37:714-7.

10. Kaplan ME, Tan EM. Antinuclear antibodies in infectious mononucleosis. Lancet 1968;291:561-3.

11. Fritzler MJ, Hanson C, Miller J, Eystathioy T. Specificity of autoantibodies to SS-A/Ro on a transfected and overexpressed human $60 \mathrm{kDa}$ Ro autoantigen substrate. J Clin Lab Anal 2002;16:103-8

12. Mahler M, Ngo JT, Schulte-Pelkum J, Luettich T, Fritzler MJ. Limited reliability of the indirect immunofluorescence technique for the detection of anti-Rib-P antibodies. Arthritis Res Ther 2008;10:R131.

13. Hamaguchi Y, Fujimoto M, Matsushita T, Kaji K, Komura K, Hasegawa M, et al. Common and distinct clinical features in adult patients with anti-aminoacyl-tRNA synthetase antibodies: heterogeneity within the syndrome. PLoS One 2013;8:e60442.

14. Cavagna L, Nuño L, Scirè CA, Govoni M, Longo FJ, Franceschini F, et al; AENEAS (American and European NEtwork of Antisynthetase Syndrome) Collaborative Group. Serum Jo-1 autoantibody and isolated arthritis in the antisynthetase syndrome: review of the literature and report of the experience of AENEAS Collaborative Group. Clin Rev Allergy Immunol 2017;52:71-80.

15. Ghirardello A, Bettio S, Bassi N, Gatto M, Beggio M, Lundberg I, et al. Autoantibody testing in patients with myositis: clinical accuracy of a multiparametric line immunoassay. Clin Exp Rheumatol 2017;35:176-7

16. Lundberg IE, Miller FW, Tjärnlund A, Bottai M. Diagnosis and classification of idiopathic inflammatory myopathies. J Intern Med 2016;280:39-51.

17. Senécal JL, Raynauld JP, Troyanov Y. Editorial: a new classification of adult autoimmune myositis. Arthritis Rheumatol 2017;69:878-84.

18. Bahmer T, Romagnoli M, Girelli F, Claussen M, Rabe KF. The use of auto-antibody testing in the evaluation of interstitial lung disease (ILD) - A practical approach for the pulmonologist. Respir Med 2016;113:80-92.

19. Kang EH, Lee EB, Shin KC, Im CH, Chung DH, Han SK, et al. Interstitial lung disease in patients with polymyositis, dermatomyositis and amyopathic dermatomyositis. Rheumatology 2005;44:1282-6.

20. Cavazzana I, Fredi M, Ceribelli A, Mordenti C, Ferrari F, Carabellese N, et al. Testing for myositis specific autoantibodies: Comparison between line blot and immunoprecipitation assays in 57 myositis sera. J Immunol Methods 2016;433:1-5.

J Rheumatol 2018;45:3; doi:10.3899/jrheum.170258 